\title{
Adults with learning disability admitted to psychiatric wards
}

\author{
Robert Chaplin \& Andrew Flynn
}

People with learning disability have an increased risk of developing a mental disorder. When they need acute psychiatric hospitalisation, they are frequently admitted to general psychiatric beds under the care of general adult psychiatrists, many of whom have had little training in the assessment and treatment of mental illness in this group. They may have unusual presentations of common mental disorders leading to difficulty in diagnosis and idiosyncratic responses to treatment. Boundary disputes between general adult and learning disability services frequently lead to a reduced quality of care for people with complex needs (see Bernal \& Hollins (1995) for an overview of psychiatric illness and learning disability). This paper will focus on the issues specific to the management of people with learning disability on general psychiatric wards, and is aimed at psychiatrists working in both general psychiatry and learning disability.

\section{Historical perspective}

Until 20 years ago, most people with learning disability who had complex needs, including mental illness, were cared for in specialist mental handicap hospitals. All medical and psychiatric care was provided within the institutions and people with learning disability did not frequently come into contact with generic services. Deinstitutionalisation has since transformed their care and in most Western countries people with learning disability now live in the community, regardless of the extent of their disabilities. This process has been guided by the principle of 'normalisation' since the early 1970s, a philosophy that remains influential today. In its simplest form, normalisation represents a statement of fundamental human rights:

"making available to all mentally retarded persons patterns of life and everyday living which are as close as possible to the regular circumstances and ways of life of society" (Nirje, 1976).

Closely associated is the concept of mainstreaming, which encourages the use of standard rather than specialised services, for example, schools, churches, shops, employment and health care. This is now firmly established as government policy that supports both the use of both generic and specialised services. The Department of Health (1992) stated that:

"wherever possible people with learning disabilities are enabled to use ordinary health services...[as well as]...specialist assessment and treatment services".

A recent working group (Royal College of Psychiatrists, 1996) acknowledged that:

"enabling people with learning disabilities to use ordinary mental health services is a complex and demanding task requiring input from specialists in the psychiatry of learning disability".

Hence, two principles of mental health care are established: joint working between general and specialist psychiatrists, and the use of generic or general psychiatric facilities where appropriate.

\section{Psychiatric admission}

Psychiatric admission may be provided by either specialist learning disability or general psychiatric

Robert Chaplin is a consultant in general adult psychiatry who works with an inner-London community mental health team (Tooting \& Furzedown CMHT, Springfield Hospital, 61 Glenburnie Road, London SW17 OJB; tel/fax: 01816826439 ) and Honorary Senior Lecturer at St George's Hospital Medical School. He has received training in learning disability at Registrar and Senior Registrar levels including a special interest session while working in Australia. Andrew Flynn is a specialist registrar in the psychiatry of learning disability. His special interests include the assertive community management of mental illness and severe challenging behaviour in adults with learning disability. 
wards. There is wide international variation in the provision of specialist services, with the UK and The Netherlands having the most developed services. In Scandinavia, the USA, Australia and most of Western Europe, few services specialise in providing mental health care and psychiatric admission is provided mainly by general psychiatric wards. In the UK, despite a long history of specialisation in learning disability psychiatry, only $70 \%$ of trusts providing learning disability services also offer specialist admission beds (Bailey \& Cooper, 1997). The debate as to whether people with learning disability should be admitted to general psychiatric wards or specialised units continues (see Box 1). The results of trials comparing outcome and satisfaction between the two systems are awaited.

\section{General adult wards for people with learning disability}

The acute admission ward has a number of advantages. Patients are admitted locally, staff may develop expertise and mainstream services are utilised, reducing stigma. Some services admit to specified beds with specially trained staff and learning disability consultant cover within general psychiatric wards (Bouras et al, 1994). In other areas, admissions occur to general psychiatric wards without specially trained staff and consultant responsibility of a general psychiatrist in liaison with the community learning disability team. Finally, patients may be admitted to general psychiatric wards by default in the absence of any specialist

\section{Box 1. Controversial issues}

General adult psychiatrists should be familiar with the assessment and treatment of people with mild learning disability and offer them admission when appropriate

General adult and learning disability psychiatrists should consult each other and agree who takes medical responsibility

Patients should not be excluded from either service following administrative disputes

Local trust protocols on psychiatric admission should be drawn up with the participation of both learning disability and general adult services

Research is needed to compare the outcomes of treatment in specialist and generic in-patient services service. The general psychiatric team assumes all responsibly for the psychiatric care in hospital and the community and the quality of such a service may be very poor (Lennox \& Chaplin, 1995).

Day (1993) highlights the disadvantages of admission to general psychiatric wards, including poor training, problems of targeting specific interventions and the rapid pace of life. Additionally, people with learning disability frequently come from protected environments, for example, family homes or residential care, and may be exposed to influences such as smoking, alcohol, illegal drugs and sexual relationships for the first time on an acute ward. They are more vulnerable to all forms of exploitation (including sexual abuse) than the general population. Furthermore, acute wards often have very high bed occupancy and a rapid turnover of patients. In such circumstances, 'bed blocking' by patients with learning disabilities may become a problem (Bouras \& Holt, 1997).

Sovner \& DesNoyers Hurley (1991) propose that seven conditions should be fulfilled in order to increase the likelihood of a good outcome from inpatient admission to a general psychiatric ward in the USA. These include: the guarantee of a discharge placement, a stable medical condition, adequate feeding and toiletting skills, the ability to communicate needs, absence of severe behavioural disorder or autism, and specific treatment goals. There should be adequate provision of appropriate activity and the use of non-pharmacological treatments.

\section{Specialist units}

A comprehensive service providing specialist services to people with the whole range of learning disability is described by Day (1993). This model has the advantages of providing an environment for a full range of therapeutic programmes, development of staff expertise, research and the containment of violent behaviour. Disadvantages are larger distances to travel and the potential for marginalisation, isolation and institutionalisation, which Day (1993) argues are not problems of well-managed hospitals. There is also the possibility of less welldeveloped assertive outreach services. Other models of specialist in-patient care include specialist wards in the grounds of psychiatric hospitals and smaller specialist units in the community. Although potentially less stigmatising and nearer their communities, they may be less able to accept patients with high levels of disturbance.

\section{Undetected learning disability}

Learning disability may not be recognised in psychiatric in-patients and may result in 
sub-optimal treatment. Learning disability may be suspected in patients with schizophrenia and cognitive impairment. Cognitive impairment is increasingly recognised in schizophrenia and performance IQ may be up to 15 points lower than verbal IQ (Nelson et al, 1990). The group of long-stay patients with chronic schizophrenia in this study had mean performance IQs of 78 with $11 \%$ below 70 . If an individual with schizophrenia is suspected of having learning disability, it is essential to enquire about his or her educational and developmental background and to estimate his or her premorbid, reading IQ and not base a diagnosis of learning disability on tests of current cognitive performance. It also follows that general psychiatric staff are probably accustomed to working with patients with cognitive deficits and could extend these skills to working with people with learning disability. The true rates of undetected learning disability in general psychiatric in-patients need further study involving careful history-taking and psychometric assessment.

\section{Training}

Many of the problems of management of people with learning disability by general psychiatrists relate to lack of training (Lennox \& Chaplin, 1995). Despite being tested at the Part II stage of the MRCPsych examinations, only a quarter of trainees receive a six-month training placement in learning disability (Carvill et al, 1999). However, from April 2000, trainees will be required to have six months' experience in either learning disability or child and adolescent psychiatry at senior house officer (SHO) level with the goal of improving understanding of developmental factors. This may also increase recruitment into specialist registrar training schemes in learning disability, some of which are currently struggling to fill their posts.

Current nurse training schemes provide an 18month common foundation training for nurses intending to apply to train in all areas of nursing. Learning disability theory is taught but placements are not common. After this period of training, student nurses training in mental health have no further opportunities to gain learning disability experience. Many senior mental health nurses receive no learning disability training at all. Lack of training may result in problems with communication and understanding, as well as negative attitudes toward people with learning disability. Similarly, student nurses in learning disability have similarly limited training in mental health, although there are newly available post-registration courses. In contrast, clinical psychologists must complete a six-month clinical placement in learning disability during their training. This runs alongside formal teaching and additional academic requirements, such as the submission of a detailed case report.

\section{Diagnosis of learning disability}

The diagnosis of learning disability requires the presence of both impairments of intelligence (usually expressed as an IQ below 70) and deficits in 'adaptive behaviour' attributable to intellectual impairment. Learning disability, in common with other diagnostic categories has a fuzzy boundary and necessarily involves a degree of social judgement. Deciding on the 'presence' or 'absence' of learning disability is a complex exercise that requires an arbitrary line to be drawn across a continuum of ability. As such, the prevalence of mild learning disability shows considerable geographical variation. Such judgements have major implications for individuals, as most countries have designed separate services for those falling on either side of their chosen cut-off. Exclusion from either adult psychiatric or learning disability services can easily result.

IQ testing itself has a controversial history. Although some forms of testing can be administered with minimal training (e.g. Raven's Progressive Matrices; Raven, 1960, 1988), the Wechsler Adult Intelligence Scales (Wechsler, 1958, 1981) are the accepted standard and have to be administered and interpreted by a clinical psychologist. Although it is tempting to base a diagnosis of learning disability on the 'full scale' alone, for reasons already discussed, it is necessary to interpret the score in context. For practical purposes, it is probably best to regard IQ score as having a correlational relationship with the clinical diagnosis of learning disability rather than providing definitive evidence.

\section{Borderline learning disability}

Borderline learning disability is a term used frequently to refer to patients with an IQ in the range 70-85 (i.e. 1-2 standard deviations below the mean). It is a category of uncertain validity and is not recognised by the ICD-10 (World Health Organization, 1992). 'Borderline intellectual functioning' appears as a V code in the DSM-IV (American Psychiatric Association, 1994). People with IQs in the range 70-85 used to be diagnosed as having mental retardation in the USA until 1971. This included $13 \%$ of the population, the majority having 
no deficits in adaptive behaviour, although Zetlin \& Murtaugh (1990) have drawn attention to the poor academic and occupational performance in people with learning disability after leaving school.

General adult psychiatrists should not exclude individuals in this group from their services. This is because only $77 \%$ of learning disability professionals indicated that they provided services to people with borderline learning disability and mental health needs (Gravestock \& Bouras, 1997). Additionally, the results of the multi-centred UK700 trial (Tyrer et al, 1999) showed that intensive case management significantly reduced the length of hospital stay for people with borderline intellectual functioning and psychosis at two-year follow-up. General adult wards may be more appropriate to their needs than specialist learning disability beds, although it is likely that they are poorly served in either setting.

\section{Mild learning disability}

Although $2 \%$ of the population have an IQ in the range 50-69, the prevalence of mild learning disability shows wide variation. Fryers (1997) cites extremes of 2.97 per 1000 in Wessex, UK, versus 77.91 per 1000 in Rose County, USA (both published in 1968). He comments on the absence of standard data and emphasises the importance of exploring the way in which the label is used across different countries.

Given the difficulty in pinning down baseline prevalence rates for mild learning disability, it is hard to be precise about its relationship to mental illness. However, there is a consensus from a range of studies in different centres that mental disorders are more common in people with mild learning disability than the wider population, with many studies observing rates between 25 and 50\% (Wilson 1997).

Despite this apparent excess of mental health needs, people with mild learning disability are prone to receive inferior care because of boundary disputes between services (Lennox \& Chaplin, 1995). Unlike people with borderline disability, those with mild disability are likely to be accepted by specialist learning disability services but may be admitted to either a specialist learning disability unit or general psychiatric ward.

\section{Assessment}

\section{General considerations}

Psychiatric assessment can be complex because of limitations in comprehension, communication and sensory deficits, as well as staff training deficits. It is important to consider that 'challenging behaviour' is frequently a symptom of a mental disorder, for example depression presenting as aggressive behaviour. In the history, the psychiatrist should pay particular attention to deterioration in patterns of behaviour, reduction in adaptive functioning and deterioration in self-care skills heralding the onset of mental illness. General psychiatrists are also likely to encounter patients with unfamiliar conditions such as autism and Asperger's syndrome and should seek specialist advice. This is particularly the case with Asperger's syndrome, regarded by some as a 'high-functioning' variant of autism where language skills are generally better developed. The ICD-10 (World Health Organization, 1992) lists guidelines for the diagnosis of both conditions.

Finally, there is the widely recognised problem of 'diagnostic overshadowing' (Reiss, 1994). This is the tendency to explain a change in emotion or behaviour in terms of learning disability rather than the expression of a comorbid mental disorder. Learning disability can be usefully seen as a vulnerability factor for the development of a mental disorder, and the psychiatrist should attempt to establish whether or not a 'dual diagnosis' is appropriate.

The relationship between learning disability and mental disorder is complex. Although 'structural' cognitive deficits (e.g. memory function and reaction times) are clearly important, it must be emphasised that this group of people have a wide experience of adverse life events and often live in impoverished social or material environments. Mild learning disability in particular shares a similar social class gradient to much mental health morbidity in the population as a whole.

\section{Psychiatric interview}

Referral is rarely at the behest of the patient and it is vital to have a flexible approach to the interview (see Box 2). History from an informant is essential and an early decision needs to be made about the appropriateness of seeing the patient or informant first or of seeing them together. The interview may require more time and can be conducted over a series of brief meetings. The atmosphere should be as relaxed and informal as possible. Talk with the patient while they are doing something enjoyable on the ward, for example, playing games. Do not be shy about using pictures or a pen and paper to help the patient communicate. Explain the purpose of the interview, and try to build a rapport by discussing an interest of the patient. Start by asking open-ended questions and proceed 
to 'either/or' questions. Be prepared to rephrase questions if they are not understood. Needless to say, care should be taken to avoid complicated terminology, talking down and the excessive use of humour (jokes that cannot be shared are not funny).

Aside from good general interviewing technique, there are some important specifics to bear in mind. Questions relating to time and frequency may not easily be understood. Acquiescence is the tendency of the interviewee to give the answer that he or she thinks the interviewer wants to hear. 'Yes/no' questions are particularly likely to have this effect, generating a series of affirmative answers. Concrete interpretation is common with the result that the interviewee may provide overly literal answers to abstract questions. Phenomena such as echolalia may also lead an interviewer to misinterpret the production of certain phrases as answers to questions. An illusion of linguistic competence can arise when someone's expressive skills are much better than his or her comprehension. In such circumstances, it is easy to miss significant impairment in communication skills.

\section{Informant interview}

The general line of enquiry is covered by Bernal \& Hollins (1995). The interviewer should enquire specifically about life events that may have precipitated the admission. The significance of bereavement is often missed (Hollins \& Esterhuysen, 1997). Events that may seem trivial can be of immense importance (for example, a change in instructor at a training centre). Obtain a history if possible from informants as well as parents, who may not be aware of sexual and relationship problems. Finally, recent research has shown that a third of cases of mental

Box 2. The psychiatric interview

Be prepared to take longer, interview on more than one occasion, and to make the interview as relaxed as possible

Interview the carer and other professionals involved (e.g. social worker, day care and residential staff)

Look carefully for life events (even if minor), bereavement and anxiety disorders, as they are often overlooked

Avoid leading questions - you may be told what they think you want to hear

'Either /or' questions are less likely to give a response set than 'yes/no' questions disorder are missed if only the patient or informant is interviewed (Moss et al, 1996). Panic disorder and phobias are particularly difficult to detect without an informant interview.

\section{Physical examination and investigations}

Physical examination assumes particular importance in cases of more severe learning disability where physical pain can be expressed in the form of 'problem behaviour' in people with limited communication skills. In addition, epilepsy in all its forms is more common in people with learning disability, and ictal phenomena (as well as the cognitive and psychiatric side-effects of anticonvulsant medication) may need to be given more weight in the psychiatric differential diagnosis. It is important to screen for abnormal movements before the prescription of antipsychotic medication.

Recent reports highlight problems in the delivery of primary care services to this group (Aspray et al, 1999) and a low take-up of preventive services such as cervical screening. Even if not considered of direct aetiological significance to mental disorder, inpatient admission offers the opportunity to review physical health and identify future services needed on discharge.

The in-patient environment affords an excellent opportunity to measure 'target behaviours'. Weight recording, monitoring of eating and measurement of social activity are useful. Mood charts (the systematic monitoring of predominant mood on each nursing shift) and sleep monitoring may give vital support to diagnosis of affective disorders. 'Problem behaviour' can be clearly defined and monitored systematically as a baseline before planning appropriate intervention.

\section{Management}

When a person with learning disability is admitted to a general psychiatric bed, the first objective is to agree on whether the general or learning disability psychiatrist acts as the responsible medical officer. It is important to recognise that admission for a person with learning disability often happens as a last resort in response to an emergency that cannot be managed elsewhere. The community learning disability team should be able to offer some training to nursing staff or even carry out defined pieces of work directly with the patient. A primary nurse with special skills or interest should be appointed in order to develop a therapeutic alliance. In some 
circumstances, a carer may need to spend a substantial amount of time assisting with personal care. The increased vulnerability of people with learning disability to abuse should be considered and protection ensured. This may need to be in the form of separation from 'high-risk' patients or an increased level of nursing observation. In all cases, close liaison with the learning disability team is essential and becomes particularly important in discharge planning. People with learning disability and mental health problems are entitled to all the provisions of the Care Programme Approach and Section 117 on after-care.

\section{Psychotropic medication}

For a good overview, see Fraser (1999).

\section{Non-pharmacological treatment}

Supportive counselling is indicated for all people with learning disability. They may need advice, explanation and reassurance about why they are in hospital, education about their illness and the buildingup of hope for recovery. For detained patients, special efforts must be made to help them understand their rights. For patients with limited verbal skills, picture books - for example, Feeling Blue (Hollins et al, 1995), which depicts a young man recovering from depression - may facilitate this process. If available, arts therapies may be particularly helpful as they can permit emotional expression for patients with problems with verbal communication. As with the management of all serious mental health problems, carers should be involved where appropriate.

Behavioural techniques to reduce 'unwanted behaviour' can be instituted following proper assessment and formulation. There are important ethical considerations concerning how such techniques are used, and readers are reminded that the Mental Health Act Code of Practice provides guidance in this area. Howlin (1997) and Emerson \& Kiernan (1997) provide excellent overviews of modern thinking in behavioural management. As with the use of medication, this is a specialist area and an appropriately trained professional should be involved in designing and monitoring behavioural interventions in conjunction with the responsible medical officer, who must sanction any such procedure.

\section{Conclusions}

Although most people with learning disability live successfully in the community, some will need periods of in-patient care. Whether this is provided by specialist or general psychiatric services depends on political factors such as the organisation of local services, as well as clinical factors, the wishes of the patient and relatives, and the level of disability. General psychiatric wards need to be flexible in their admission policies as many individuals with learning disability may be more appropriately managed in that environment. General psychiatrists, therefore, need to possess the clinical skills to assess and manage mental disorders in people with learning disability, in liaison with psychiatrists in learning disability. Areas of unmet need should be identified and purchasers made aware of deficiencies. Changes to the training of $\mathrm{SHOs}$ are likely to result in an increase in expertise in the psychiatry of learning disability in general psychiatrists in the future.

\section{References}

American Psychiatric Association (1994) Diagnostic and Statistical Manual of Mental Disorders (4th edn) (DSM-IV). Washington, DC: APA.

Aspray, T. J., Francis, R. M., Tyrer, S. P., et al (1999) Patients with learning disability in the community: have special needs that should be planned for. Editorial. British Medical Journal, 318, 476-477

Bailey, N. M. \& Cooper, S.-A. (1997) The current provision of specialist health services to people with learning disabilities in England and Wales. Journal of Intellectual Disability Research, 41, 52-59.

Bernal, J. \& Hollins, S. (1995) Psychiatric illness and learning disability: a dual diagnosis. Advances in Psychiatric Treatment, 1, 138-145.

Bouras, N., Brooks, D. \& Drummond, K. (1994) Community services for people with mental retardation. In Mental Health in Mental Retardation: Recent Advances and Practices, (ed. N. Bouras), pp. 293-299. Cambridge: Cambridge University Press.

Bouras, N. \& Holt, G. (1997) Crisis in London's mental health services. Meeting the needs of people with learning disabilities would unblock acute beds (letter). British Medical Journal, 314, 1278-1279.

Carvill, S., Marston, G. \& Hollins, S. (1999) Trainee attitudes within the Faculty of the Psychiatry of Learning Disability. Psychiatric Bulletin, 23, 86-89.

Day, K. A. (1993) Mental health services for people with mental retardation: a framework for the future. Journal of Intellectual Disability Research, 37, 7-16.

Department of Health (1992) Health Services for People with Learning Disabilities (Mental Handicap). HSG(92)42. London: Department of Health.

Emerson, E. \& Kiernan, C. (1997) Behavioural interventions In Seminars in the Psychiatry of Learning Disabilities (ed. O. Russell), pp. 161-178. London: Gaskell.

Fraser, B. (1999) Psychopharmacology and people with learning disability. Advances in Psychiatric Treatment, $\mathbf{5}$ $471-477$.

Fryers, T. (1997) Impairment, disability and handicap: categories and classifications. In Seminars in the Psychiatry of Learning Disabilities (ed. O. Russell), pp. 16-30. London: Gaskell.

Gravestock, S. \& Bouras, N. (1997) Survey of services for adults with learning disabilities. Psychiatric Bulletin, 21, 197-199. 
Hollins, S. \& Esterhuyzen, A. (1997) Bereavement and grief in adults with learning disabilities. British Journal of Psychiatry, 170, 479-501.

—, Curran J. \& Webb, B. (1995) Feeling Blue. London: St George's Mental Health Library.

Howlin, P. (1997) Interventions for people with autism: recent advances. Advances in Psychiatric Treatment, 3, 94-102.

Lennox, N. \& Chaplin, R. H. (1995). Intellectual disability: the views of psychiatric trainees. Australian and New Zealand Journal of Psychiatry, 29, 632-637.

Moss, S., Prosser, H., Ibbotson, B., et al (1996) Respondent and informant accounts of psychiatric symptoms in a sample of patients with mild learning disability. Journal of Intellectual Disability Research, 40, 457-465.

Nelson, H. E., Pantelis, C., Carruthers, K., et al (1990) Cognitive functioning and symptomatology in chronic schizophrenia. Psychological Medicine, 20, 357-365.

Nirje, B. (1976) The normalisation principle and its human management implications. In Normalisation, Social Integration and Community Services (eds R. J. Flynn \& K. E. Nitsch). Baltimore, MD: University Park Press.

Raven, J. C. (1960) Guide to the Standard Progressive Matrices. London: H. K. Lewis.

- (1988) Raven's Progressive Matrices and Vocabulary Scales. Oxford: Oxford University Press.

Reiss, S. (1994) Psychopathology in mental retardation. In Mental Health in Mental Retardation: Recent Advances and Practices (ed. N. Bouras), pp. 67-78. Cambridge: Cambridge University Press.

Royal College of Psychiatrists (1996) Meeting the Mental Health Needs of People with Learning Disability. Council Report CR56. London: Royal College of Psychiatrists.

Sovner, R. \& DesNoyers Hurley, A. (1991) Seven questions to ask when considering an acute psychiatric inpatient admission for a developmentally disabled adult. Habilitative Mental Healthcare Newsletter, 10, 27-30.

Tyrer, P., Hassiotis, A., Ukoumunne, O., et al for the UK 700 Group (1999) Intensive case management for psychotic patients with borderline intelligence. Lancet, 354, 999-1000.

Wechsler, D. (1958) The Measurement and Appraisal of Adult Intelligence (4th edn). Baltimore, MD: Williams \& Wilkins.

-_- (1981) WAIS-R Manual. New York: Psychological Corporation.

Wilson, D. (1997) Psychiatric disorders and mild learning disability. In Seminars in the Psychiatry of Learning Disability. (ed. O. Russell), pp. 125-135. London: Gaskell.

World Health Organization (1992) The ICD-10 Classification of Mental And Behavioural Disorders. Geneva: WHO.

Zetlin, A. \& Murtaugh, M. (1990) Whatever happened to those with borderline IQs? American Journal on Mental Retardation, 94, 463-469.

\section{Multiple choice questions}

1. Borderline learning disability:

$a$ is recognised by the ICD-10

$\mathrm{b}$ is a category of disputed validity

c affects $13 \%$ of the adult population

$\mathrm{d}$ is usually associated with deficits in adaptive behaviour

e the majority of services for people with learning disability are likely to accept adults with IQs in this range.

2. Acceptable models of in-patient care for adults with mild learning disability include:

a acute treatment wards in a specialist learning disability hospital

b specialist learning disability wards in the community

c general adult wards with specially designated beds under care of a consultant in learning disability psychiatry

d general adult wards under the care of a general psychiatrist but in close liaison with the learning disability team

e general adult wards with no liaison from specialist learning disability staff.

3. When interviewing people with learning disability:

a yes/no questions are better than either/or questions

$\mathrm{b}$ the interview should begin with leading questions

c the use of a pen and paper or a picture book can be helpful

$\mathrm{d}$ the patient should only be interviewed with his or her parents or carer present

e diagnostic overshadowing is a useful interview technique.

4. Research findings have shown in people with learning disability:

a admission to a specialist learning disability ward is superior to a general psychiatric ward

b assertive outreach may successfully prevent psychiatric admission

c the majority of trusts have specialist learning disability in-patient wards

d atypical antipsychotics may be particularly helpful in the management of autism

e the prevalence of mild learning disability is broadly similar in different countries.

MCQ answers

$\begin{array}{lllll}\text { 1 } & \text { 2 } & 3 & 4 \\ \text { a F } & \text { a T } & \text { a F } & \text { a F } \\ \text { b T } & \text { b T } & \text { b F } & \text { b T } \\ \text { c T } & \text { c T } & \text { c T } & \text { c T } \\ \text { d F } & \text { d T } & \text { d F } & \text { d T } \\ \text { e T } & \text { e F } & \text { e F } & \text { e F }\end{array}$

